\title{
ALGUNS ASPECTOS REFERENTES AO ESTUDO DE LINHAGENS DE SCHISTOSOMA MANSONI SAMBON, 1907, PROVENIENTES DOS ESTADOS DE MINAS GERAIS E DE SÃO PAULO, BRASIL*.
}

Raimundo Carlos Lemos Neto $* *$

Luiz Augusto Magalhães ***:

Aquiles Eugênico Piedrabuena ***

\begin{tabular}{r|r|} 
RSPUB9/416 \\
\hline
\end{tabular}

Lemos Neto, R. C. et al. Alguns aspectos referentes ao estudo de linhagens de Schistosoma mansoni Sambon, 1907, provenientes dos Estados de Minas Gerais $e$ de São Paulo, Brasil. Rev. Saúde públ., S. Paulo, 12:277-90, 1978.

RESUMO: Foram estudados alguns aspectos do comportamento das linhagens mineira (BH) e paulista (SJ) do S. mansoni. Pesquisou-se a capacidade de penetração das cercárias de ambas as linhagens e a relaçáo granulomas hepáticosvermes. Verificoul-se que as cercárias da linhagem $B H$ possuem maior capacidade de penetração e que o número de granulomas hepáticos por verme, nas dalas linhagens, variou inversamente com o numero de vermes por camundongo.

Uniteruos: Schistosoma mansoni, linhagens. Esquistossomose mansonica.

\section{T T RODUC AO}

Vários experimentos já demonstraram diferenças no comportamento biológico entre as linhagens de $S$. mansoni de Belo Horizonte, $M G$ (Linhagem $B H$ ) e do Vale do Rio Paraíba do Sul, SP (linhagem SJ). Assim é que desde 1963, quando Paraense e Corrêa ${ }^{12}$ assinalaram a existência destas linhagens, vários outros autores acrescentaram dados aos estudos destas cepas. Trabalhos de Magalhães e Carvalho T.9.1" (1969, 1973 e 1976) e Magalhães e col.11 (1975) assinalaram que estas linhagens di- ferem quanto à capacidade de penetração das cercárias; a alguns aspectos morfológicos dos esquistossomos adultos; e à atividade patogênica no camundongo de laboratório.

No presente trabalho propusemo-nos reestudar alguns destes aspectos, concentrando nossa atenção na avaliação da influência do numero de vermes adultos por camundongo sobre a relação granuloma hepático por verme.

\footnotetext{
* Trabalho realizado no Instituto de Biologia da Universidade Estadual de Campinas (UNICAMP).

Do Departamento de Microbiologia e Parasitologia da Universidade do Maranhão - Largo dos Amores, 66 - 65000 - São Luís, MA - Brasil.

Do Instituto de Biologia da UNICAMP - Caixa Postal 1170 - 13100 - Campinas. SP Brasil.
} 
LEMOS NETO, R. C. et al. Alguns aspectos referentes ao estudo de linhagens de Schistosoma mansoni Sambon, 1907, provenientes dos Estados de Minas Gerais e de São Paulo, Brasil. Rev. Saúde pübl., S. Paulo, 12:277-90, 1978.

MATERIAL E METODOS

Utilizamos em nossas pesquisas, cercárias procedentes de Biomphalaria glabrata de Belo Horizone, MG (linhagem BH) e de Biomphalaria tenagophila do Vale do Rio Paraíba do Sul, SP (linhagem SJ).

Para a obtenção das cercárias foi adotada a técnica preconizada por Pellegrino e Macedo ${ }^{14}$ (1955).

Com as cercárias obtidas, infectamos 60 camundongos fêmeas, pesando, aproximadamente, $16 \mathrm{~g}$. Os roedores assim infectados foram divididos em dois lotes de 30 , levando-se em consideração a procedência das cercárias: lote 1 (linhagem $\mathrm{BH}$ ) e lote 2 (linhagem SJ).

Os lotes 1 e 2 foram subdivididos, adotando-se por critério o número de cercárias infectantes, conforme abaixo relacionado:

Lote 1: sub lote $1 \mathrm{~A}-15$ camundongos infectados, cada um, com 100 cercárias da linhagem $\mathrm{BH}$

sub lote $1 \mathrm{~B}-15$ camundongos infectados, cada um, com 200 cercárias da linhagem BH

Lote 2: sub lote $2 \mathrm{~A}-15$ camundongos infectados, cada um, com 100 cercárias da linhagem $\mathrm{SJ}$

sob lote $2 B$ - 15 camundongos infectados, cada um, com 200 cercárias da linhagem $\mathrm{S}$ J

Paralelamente a esta experiência, infectou-se dois outros grupos de 15 camundongos (grupos $\mathrm{C}$ e $\mathrm{D}$ ), respectivamente, de 200 cercárias de $\mathrm{BH}$ e SJ, com a finalidade de determinarmos a viabilidade de penetração das cercárias.

A infecção dos camundongos, pertencentes aos lotes 1 e 2 e aos grupos C e D, foi feita por imersão da cauda (Magalhães ${ }^{6}$, 1969). As cercárias foram utilizadas 2 hs após o início da exposição dos moluscos à luz e ao calor.
Decorridos sessenta dias da data da infecção, os camundongos pertencentes aos lotes 1 e 2 foram necropsiados e o seu sistema porta perfundido (Yolles e col.17, 1947). Além de contarmos os vermes obtidos por perfusão do sistema porta, contamos também os esquistossomos localizados no fígado dos roedores (Standen ${ }^{16}$, 1953 e Hill 5, 1956).

Para a contagem de granulomas utilizamos a técnica de Pellegrino e Brener ${ }^{13}$ (1956) e Brener e col. ${ }^{3}$ (1956).

\section{RESULTADOS}

Aos sessenta dias após a exposição cercariana, contamos os vermes e os granulomas hepáticos obtidos por necropsia, nos camundongos dos lotes 1 e 2 (Tabelas 1 e 2). Verificamos que a média de vermes por camundongo na linhagem $\mathrm{BH}$ foi inferior à média equivalente da linhagem SJ, tanto para camundongos expostos a 100 como a 200 cercárias. Entretanto, na linhagem mineira os valores referentes à relação granuloma hepático por verme foram mais elevados (Tabela 2).

Com os valores correspondentes ao total de granulomas hepáticos e vermes por camundongos (Tabela 1), realizamos estudos estatísticos comparativos entre as duas linhagens de $S$. mansoni. $O$ número de vermes adultos e de granulomas hepáticos obtidos em cada camundongo perfundido foi ordenado em ordem decrescente de granulomas (Tabelas 3 e 4).

A correlação encontrada entre as linhagens BH e SJ está exposta na Tabela 5.

Utilizando os valores apresentados pelas relações granulomas hepáticos-vermes, contidos nas Tabelas 3 e 4 , avaliamos a função $G H=f(H M)$ para $B H$ e SJ, onde $\mathrm{GH}$ corresponde ao número de granulomas hepáticos por camundongo e $\mathrm{HM}$ ao número total de vermes por camundongo. As equaçōes estabelecidas para o ajustamento linear estão transcritas abaixo e representadas na Fig. 1. 
LEMOS NETO, R. C. et al. Alguns aspectos referentes ao estudo de linhagens de Schistosoma mansoni Sambon, 1907, provenientes dos Estados de Minas Gerais e de São Paulo, Brasil. Rev. Saúde públ., S. Paulo, 12:277-90, 1978.

T A B E L A 1

Vermes e granulomas obtidos da perfusão de 48 camundongos infectados pela linhagem BH e SJ. Dados obtidos ap6́s 60 dias de infecção.

\begin{tabular}{|c|c|c|c|c|c|}
\hline \multirow[b]{2}{*}{ Linhagem } & \multirow[b]{2}{*}{$\begin{array}{l}\text { Número de } \\
\text { camundongos* }\end{array}$} & \multirow[b]{2}{*}{$\begin{array}{l}\text { Cercárias por } \\
\text { camundongo }\end{array}$} & \multicolumn{2}{|c|}{ Vermes } & \multirow[b]{2}{*}{$\begin{array}{c}\text { Granulomas } \\
\text { hepáticos }\end{array}$} \\
\hline & & & $\begin{array}{c}\text { Machos } \\
\text { e } \\
\text { Fêmeas }\end{array}$ & Fêmeas & \\
\hline \multirow{3}{*}{$\mathrm{BH}$} & 12 & 100 & 88 & 34 & 19.706 \\
\hline & 12 & 200 & 113 & 46 & 25.794 \\
\hline & & Totais & 201 & 80 & 45.500 \\
\hline \multirow{3}{*}{$\mathbf{S J}$} & 12 & 100 & 144 & 41 & 20.201 \\
\hline & 12 & 200 & 217 & 59 & 31.210 \\
\hline & & Totais & 361 & 100 & 51.411 \\
\hline
\end{tabular}

* sobreviventes

T A B E LA 2

Média de vermes por camundongo e de granulomas hepáticos por verme das linhagens BH e SJ

\begin{tabular}{ccccc}
\hline Linhagem & $\begin{array}{c}\text { Cercárias por } \\
\text { camundongo }\end{array}$ & $\begin{array}{c}\text { Vermes por } \\
\text { camundongo }\end{array}$ & \multicolumn{2}{c}{$\begin{array}{c}\text { Granulomas hepáticos } \\
\text { por verme }\end{array}$} \\
\hline BH & 100 & $\begin{array}{c}\text { Machos e } \\
\text { Fêmeas }\end{array}$ & Fêmeas \\
\hline SJ & 200 & 7,3 & 580 & 561 \\
\hline & 100 & 12,0 & 140 & 493 \\
\hline
\end{tabular}

$\mathrm{BH} y=118,49329 \cdot x+902,95202$

SJ $y^{1}=136,12317 \cdot x+94,60562$

Aplicamos o teste $B$ de significância (Piedrabuena e Baracho ${ }^{15}$, 1976), entre os coeficientes de correlação encontrados para BH e SJ, de acordo com a seguinte fórmula:

$$
B=\frac{\left(1+r_{1}\right)\left(1-r_{2}\right)}{\left(1+r_{2}\right)\left(1-r_{1}\right)} \text { sendo }
$$

$$
\begin{aligned}
& r_{1}=0,84994 \\
& r_{2}=0,82343
\end{aligned}
$$

$\mathrm{B}=1,194<3,35$ (valor de $\mathrm{B}$ da Tabela, ao nivel de $5 \%$ ) N.S. sendo $n_{1}=n_{2}$ (número de casos) $=24$. 
T A B E L 3

Relação dos granulomas hepáticos por camundongo e número de granulomas por verme da linhagem $\mathrm{BH}$.

\begin{tabular}{|c|c|c|c|c|}
\hline $\begin{array}{l}\text { Número de } \\
\text { granulomas por } \\
\text { camundongo }\end{array}$ & $\begin{array}{l}\text { Vermes } \\
\text { Machos }\end{array}$ & $\begin{array}{l}\text { Vermes } \\
\text { Fêmeas }\end{array}$ & $\begin{array}{l}\text { Total de } \\
\text { vermes por } \\
\text { camundongo }\end{array}$ & $\begin{array}{l}\text { Número de } \\
\text { granulomas } \\
\text { por verme }\end{array}$ \\
\hline 3077 & 10 & 7 & 17 & 181 \\
\hline 3000 & 10 & 6 & 16 & 187 \\
\hline 2923 & 9 & 6 & 15 & 195 \\
\hline 2659 & 8 & 6 & 14 & 190 \\
\hline 2505 & 8 & 6 & 14 & 179 \\
\hline 2319 & 7 & 5 & 12 & 193 \\
\hline 2319 & 6 & 5 & 11 & 211 \\
\hline 2242 & 6 & 5 & 11 & 204 \\
\hline 2154 & 8 & 2 & 10 & 215 \\
\hline 2142 & 2 & 4 & 6 & 357 \\
\hline 2077 & 7 & 5 & 12 & 173 \\
\hline 1955 & 3 & 3 & 6 & 326 \\
\hline 1911 & 3 & 3 & 6 & 319 \\
\hline 1857 & 5 & 3 & 8 & 232 \\
\hline 1857 & 4 & 2 & 6 & 309 \\
\hline 1714 & 3 & 2 & 5 & 343 \\
\hline 1549 & 2 & 2 & 4 & 387 \\
\hline 1385 & 3 & 1 & 4 & 346 \\
\hline 1252 & 1 & 2 & 3 & 417 \\
\hline 1049 & 2 & 1 & 3 & 350 \\
\hline 1049 & 2 & $\mathbf{1}$ & 3 & 350 \\
\hline 1011 & 2 & 1 & 3 & 337 \\
\hline 879 & - & 1 & 1 & 879 \\
\hline 615 & 10 & 1 & 11 & 56 \\
\hline Total 45.500 & 121 & 80 & 201 & 6.936 \\
\hline
\end{tabular}

T A B E L A 4

Relação dos granulomas hepáticos por camundongo e número de granulomas por vermes da linhagem $\mathrm{SJ}$.

\begin{tabular}{|c|c|c|c|c|}
\hline $\begin{array}{l}\text { Número de } \\
\text { granulomas por } \\
\text { camundongo }\end{array}$ & $\begin{array}{l}\text { Vermes } \\
\text { Machos }\end{array}$ & $\begin{array}{l}\text { Vermes } \\
\text { Fêmeas }\end{array}$ & $\begin{array}{l}\text { Total de } \\
\text { vermes por } \\
\text { camundongo }\end{array}$ & $\begin{array}{l}\text { Número de } \\
\text { granulomas } \\
\text { por verme }\end{array}$ \\
\hline 6209 & 38 & 6 & 44 & 141 \\
\hline 4813 & 19 & 15 & 34 & 141 \\
\hline 4434 & 17 & 13 & 30 & 147 \\
\hline 3616 & 13 & 9 & 22 & 164 \\
\hline 3626 & 15 & 7 & 22 & 165 \\
\hline 3253 & 14 & 6 & 20 & 163 \\
\hline 3186 & 13 & 6 & 19 & 168 \\
\hline 3186 & 8 & 2 & 10 & 319 \\
\hline 3109 & 16 & 4 & 20 & 155 \\
\hline 2746 & 5 & 6 & 11 & 250 \\
\hline 2163 & 12 & 3 & 15 & 144 \\
\hline 1760 & 5 & 4 & 9 & 195 \\
\hline 1714 & 8 & 2 & 10 & 171 \\
\hline 1659 & 8 & 2 & 10 & 166 \\
\hline 1494 & 5 & 2 & $T$ & 213 \\
\hline 1428 & 4 & 2 & 6 & 238 \\
\hline 875 & 16 & 3 & 19 & 46 \\
\hline 615 & 17 & 2 & 19 & 32 \\
\hline 417 & 4 & 1 & 5 & 83 \\
\hline 406 & 3 & 1 & 4 & 101 \\
\hline 340 & 2 & 1 & 3 & 113 \\
\hline 154 & 2 & 3 & 5 & 31 \\
\hline 154 & 3 & - & 3 & 51 \\
\hline 154 & 14 & - & 14 & 11 \\
\hline Total 51.411 & 261 & 100 & 361 & 3408 \\
\hline
\end{tabular}


LEMOS NETO, R. C. et al. Alguns aspectos referentes ao estudo de linhagens de Schistosoma mansoni Sambon, 1907, provenientes dos Estados de Minas Gerais e de São Paulo, Brasil. Rev. Saúde públ., S. Paulo, 12:277-90, 1978.

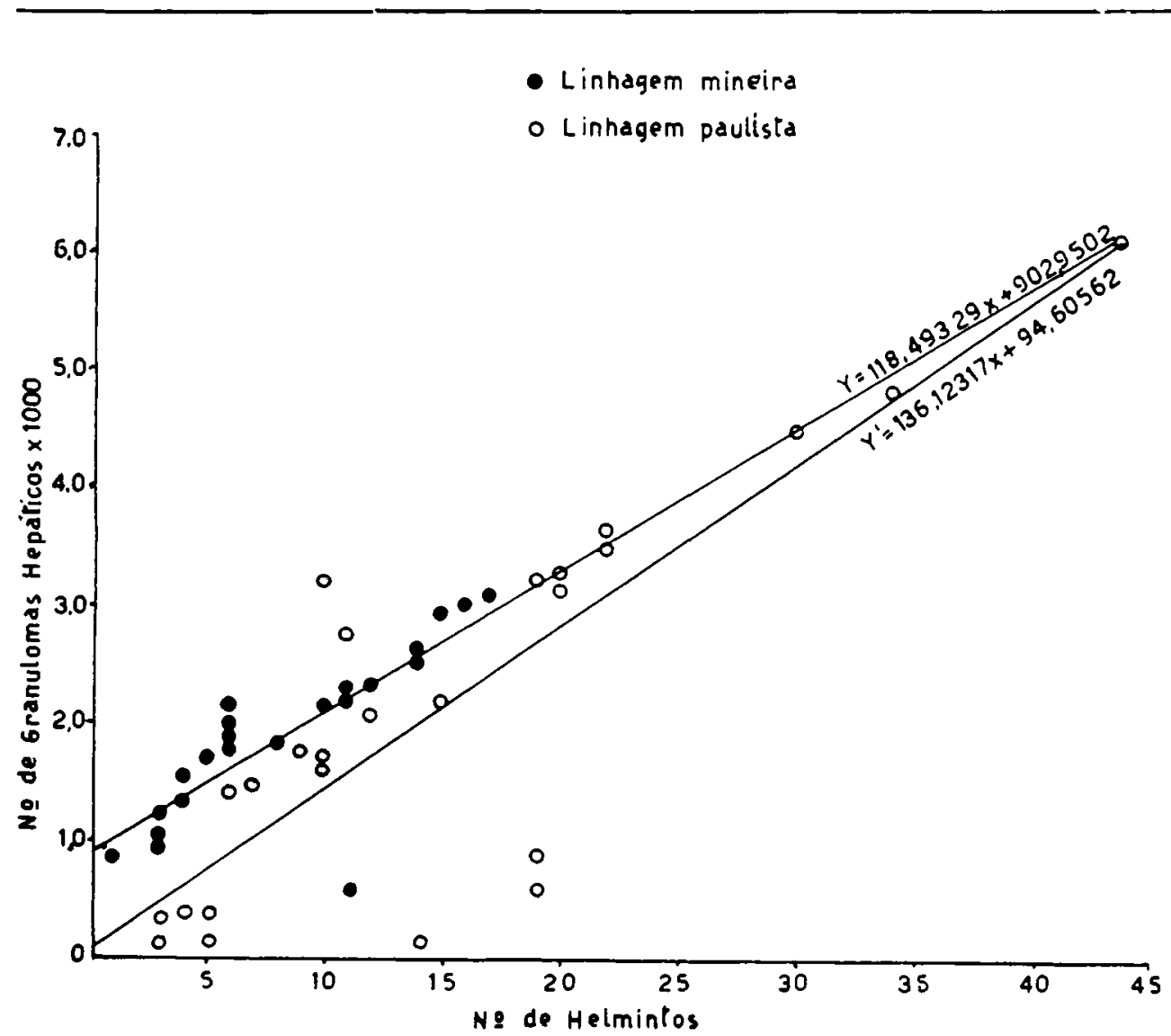

Fig. 1 - Relação entre granulomas hepáticos $\epsilon$ número de vermes adultos das linhagens $\mathrm{BH}$ e SJ encontrados em Mus musculus.

T A B E L A 5

Relação granulomas hepáticos/vermes: coeficientes de correlação, regressão e termo independente.

\begin{tabular}{llllc}
\hline & & (BH) & (SJ) \\
\hline Coef. de correlação & (r) & $0,82343^{* * *}$ & $0,84994^{* * *}$ \\
Coef. de regressão & (b) & 118,49329 & 136,12317 \\
Termo independente & (a) & 902,95202 & 94,60562 \\
\hline
\end{tabular}

*** Significativos ao nível de 0,001 
LEMOS NETO, R. C. et al. Alguns aspectos referentes ao estudo de linhagens de Schistosoma mansoni Sambon, 1907, provenientes dos Estados de Minas Gerais e de São Paulo, Brasil. Rev. Saúde públ., S. Paulo, $12: 277-90,1978$.

Para o ajustamento hiperbólico, consideramos os dados das Tabelas 3 e 4 , destituidas dos valores 615 e 11 de $\mathrm{BH}$ e 154 e 14 de SJ, por estarem muito afastados da média dos valores encontrados. Os valores ajustados, tomados para a função hiperbólica, estão contidos na Tabela 6 e obedecem as equações:

$$
\begin{aligned}
& \text { BH } y=798,52 \cdot X^{0.44415} \\
& \text { SJ } y^{1}=85,96 \cdot X^{1,15879}
\end{aligned}
$$

Estes valores encontram-se nas Figs. 2 e 3 , representativos das funçōes $\mathrm{GH}=\mathrm{f}$ (HM).

Nos estudos comparativos entre $\mathrm{BH}$ e SJ, consideramos, ainda, o relacionamento entre o total de vermes por camundongo e o número de granulomas hepáticos produzidos por verme (Tabelas 3 e 4). Não incluímos nos cálculos estatísticos os valores constantes da última linha da Tabela 3 , por considerá-los muito afastados dos outros valores assinalados.

T A B E LA B

Valores referentes ao ajustamento hiperbólico da relação granulomas hepáticos por camundongo/

\begin{tabular}{|c|c|c|c|c|c|}
\hline & Linhagem $\mathbf{B H}$ & & & Linhagem $\mathrm{SJ}$ & \\
\hline $\begin{array}{l}\text { Total de } \\
\text { vermes por } \\
\text { camundongo } \\
(\mathrm{X})\end{array}$ & $\begin{array}{c}\mathrm{N}^{\circ} \text { de } \\
\text { granulomas } \\
\text { por camundongo } \\
(\mathrm{Y})\end{array}$ & $\begin{array}{l}\text { Valores } \\
\text { ajustados } \\
\text { para } Y \\
\left(Y_{C}\right)\end{array}$ & $\begin{array}{c}\text { Total de } \\
\text { vermes por } \\
\text { camundongo } \\
\text { (X) }\end{array}$ & $\begin{array}{l}\text { No de } \\
\text { granulomas por } \\
\text { camundongo } \\
\text { (Y) }\end{array}$ & $\begin{array}{l}\text { Valores } \\
\text { ajustados } \\
\text { para } \mathrm{Y} \\
(\mathrm{Yc})\end{array}$ \\
\hline 17 & 3077 & 2811 & 44 & 6209 & 6888 \\
\hline 16 & 3000 & 2734 & 34 & 4813 & 5116 \\
\hline 15 & 2923 & 2659 & 30 & 4434 & 4426 \\
\hline 14 & 2659 & 2578 & 22 & 3616 & 3089 \\
\hline 14 & 2505 & 2578 & 22 & 3526 & 3089 \\
\hline 12 & 2319 & 2408 & 20 & 3253 & 2767 \\
\hline 11 & 2319 & 2316 & 19 & 3186 & 2606 \\
\hline 11 & 2242 & 2316 & 10 & 3186 & 1239 \\
\hline 10 & 2154 & 2220 & 20 & 3109 & 2767 \\
\hline 6 & 2142 & 1770 & 11 & 2746 & 1384 \\
\hline 12 & 2077 & 2408 & 15 & 2163 & 1982 \\
\hline 6 & 1955 & 1770 & 9 & 1760 & 1096 \\
\hline 6 & 1911 & 1770 & 10 & 1714 & 1239 \\
\hline 8 & 1857 & 2011 & 10 & 1659 & 1239 \\
\hline 6 & 1857 & 1770 & 7 & 1494 & 820 \\
\hline 5 & 1714 & 1632 & 6 & 1428 & 685 \\
\hline 4 & 1549 & 1478 & 19 & 875 & 2606 \\
\hline 4 & 1385 & 1478 & 19 & 615 & 2606 \\
\hline 3 & 1252 & 1301 & 5 & 417 & 555 \\
\hline 3 & 1409 & 1301 & 4 & 406 & 429 \\
\hline 3 & 1049 & 1301 & 3 & 340 & 307 \\
\hline 3 & 1011 & 1301 & 5 & 154 & 192 \\
\hline 1 & 879 & 798 & 3 & 154 & 86 \\
\hline
\end{tabular}
vermes por camundongo das linhagens de Belo Horizonte e do Vale do Rio Paraíba. 
LEMOS NETO, R. C. et al. Alguns aspectos referentes ao estudo de linhagens de Schistosoma mansoni Sambon, 1907, provenientes dos Estados de Minas Gerais e de São Paulo, Brasil. Rev. Saúde públ., S. Paulo, 12:277-90, 1978.

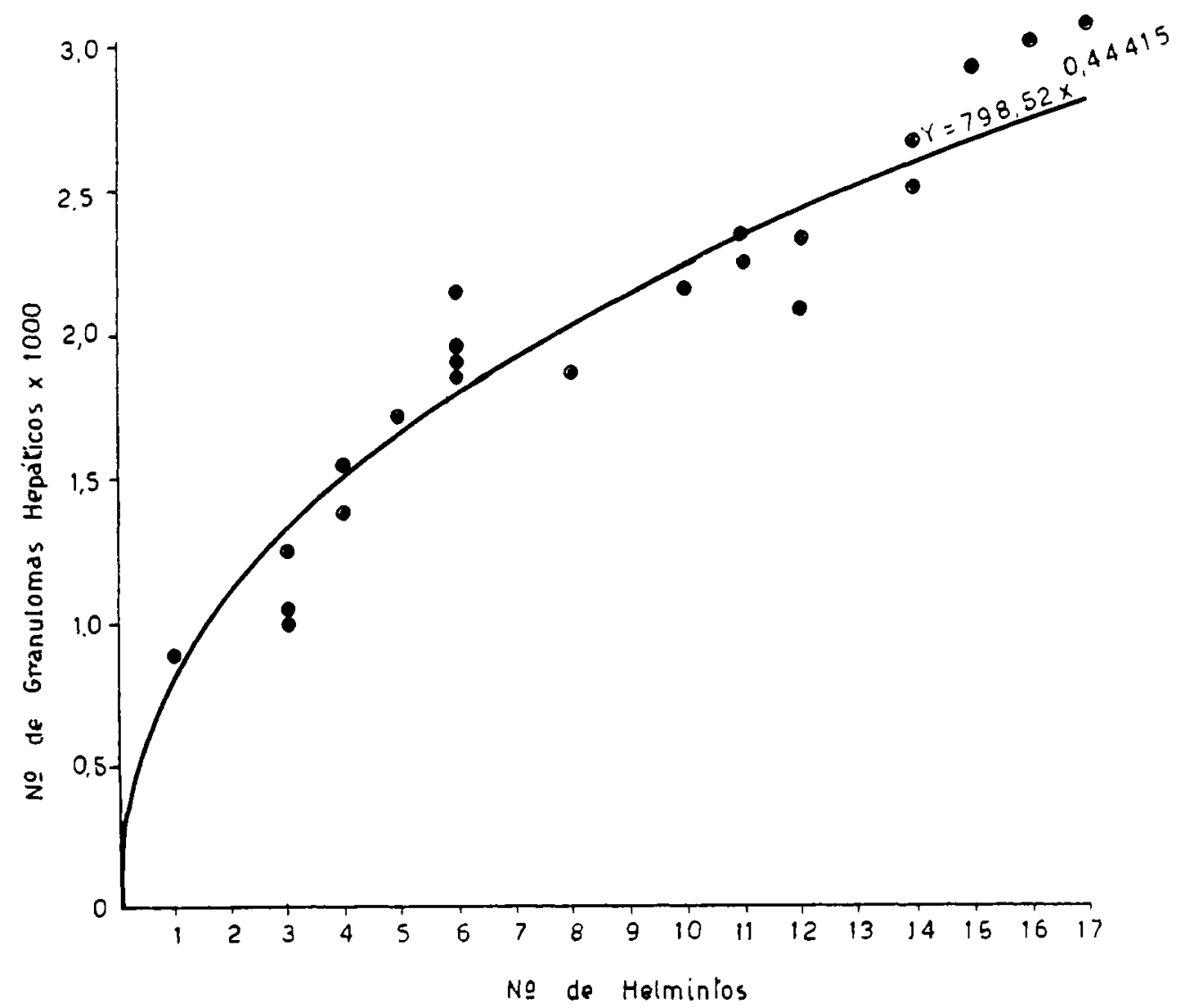

Fig. 2 - Relação entre granulomas hepáticos e número de vermes adultos da linhagem BH encontrados em Mus musculus.

Os dados da Tabela 4 apresentan dois grupos distintos de distribuição. Avaliamos a função $G H=f(H M)$ em separado, para estes dois tipos de valores que correspondem a SJI e SJ2, abaixo especificados.

Os valores ajustados, para as linhagens mineira $(\mathrm{BH})$ e paulista ( $\mathrm{S} J)$, acham-se na Tabela 7 e obedecem as equações abaixo:

BH y $=376,28 . X^{-0 ; 51835}$

SJ1 $y^{\prime}=458,610 . X^{-0,34445}$

SJ2 $y^{\prime \prime}=206,076 \cdot X^{-0,59498}$
Estas funçöes encontram-se representadas na Fig. 4.

Os resultados referentes ao número de cercárias que penetraram no tegumento da cauda de Mus musculus albinos, pertencentes aos lotes C (linhagem BH) e D (linhagem SJ), encontram-se dispostos na Tabela 8. Estes dados foram analisados estatisticamente, utilizando-se somente o número de cercárias que não penetraram. Devido à heterogeneidade dos dados (os $\mathrm{X}^{2}$ obtidos foram $232,263 *$ e $28,049 *$ com 13 graus de

\footnotetext{
* Significativo ao nivel de 0,05
} 
LEMOS NETO, R. C. et al. Alguns aspectos referentes ao estudo de linhagens de Schistosoma mansoni Sambon, 1907, provenientes dos Estados de Minas Gerais e de São Paulo, Brasil. Rev. Saude públ., S. Paulo, 12:277-90, 1978.

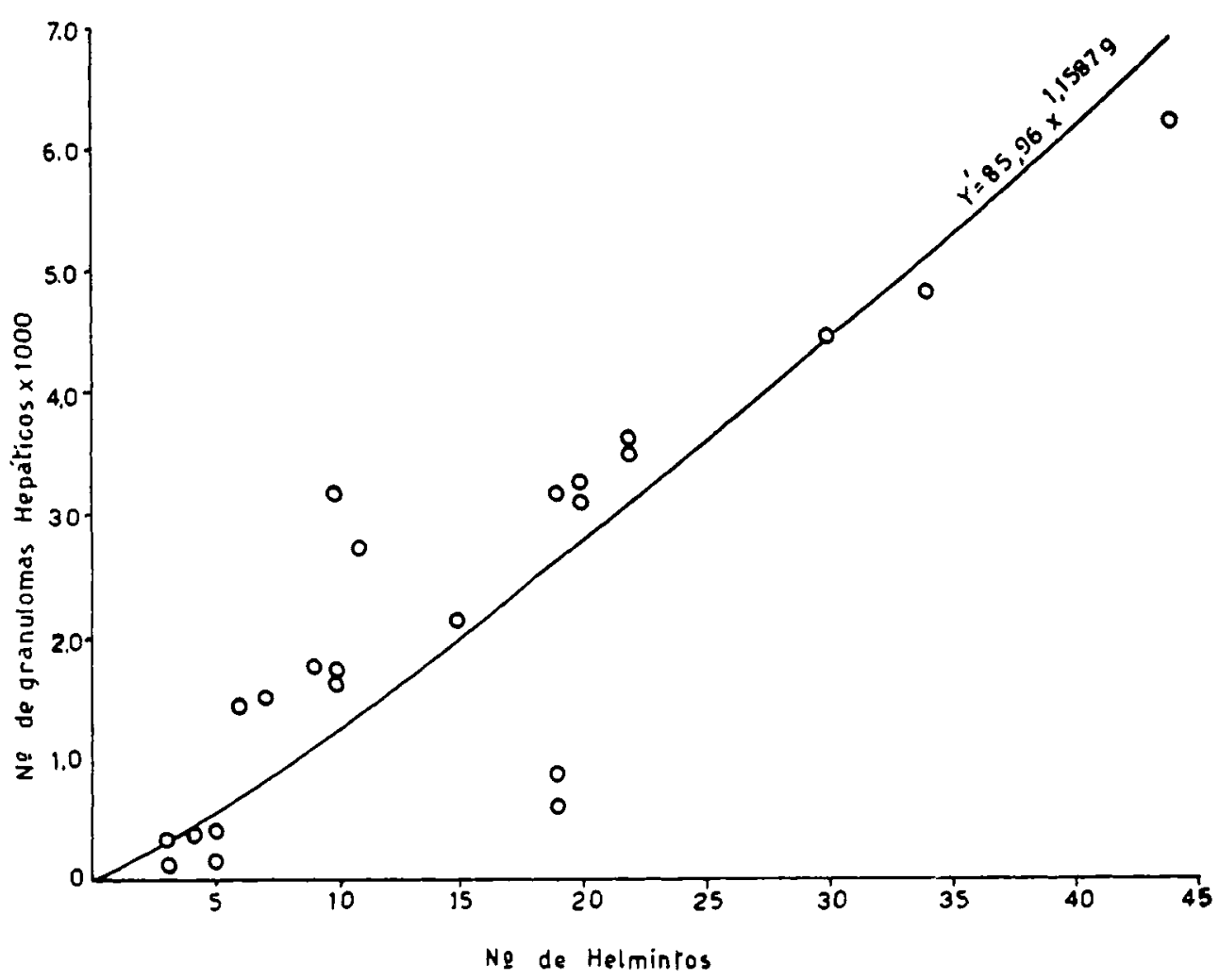

Fig. 3 - Relaçăo entre granulomas hepáticos e número de vermes adultos de linhagem $\mathbf{S J}$ encontrados em Mus musculus.

liberdade para as linhgens $\mathrm{BH}$ e SJ, respectivamente), exprimimo-los em percentagem e os valores foram transformados em ângulos, utilizando-se, para isso, a fórmula $\alpha^{0}=\operatorname{arco}$ seno $\sqrt{\mathrm{P}}$ (Ficher e Yates 4,1971 ) (Tabela 9). Estudadas as linhagens por análise de variância, elas representaram um valor de $F$ igual a 112,879 , altamente significativo com 1 e 28 graus de liberdade. Encontramos um coeficiente de variabilidade igual a $13,46 \%$. Os dados referentes à mortalidade observada nos lotes de camundongos 1 e 2 estão apresentados na $\mathrm{Ta}$ bela 10 .

\section{DISCUSSÃO E CONCLUSAO}

A mortalidade encontrada nas duas populações de camundongos infectados pelas linhagens assinaladas foi praticamente a mesma (Tabela 10). Como será descrito adiante, a linhagem mineira mostrou-se mais patogênica.

Em vista deste fato, era de se esperar uma maior letalidade nos grupos de animais infectados por esta cepa. Como o numero de vermes encontrados nos camundongos infectados pela linhagem mineira foi muito menor do que os obtidos nos 
LEMOS NETO, R. C. et al. Alguns aspectos referentes ao estudo de linhagens de Schistosoma mansoni Sambon, 1907, provenientes dos Estados de Minas Gerais e de São Paulo, Brasil. Rev. Saúde pübl,. S. Paulo, 12:277-90, 1978.

\section{T A B EL A 7}

Valores referentes ao ajustamento hiperbólico da relação vermes por camundongo/número de granulomas por verme das linhagens de Belo Horizonte e do Vale do Rio Paraíba.

\begin{tabular}{|c|c|c|c|c|c|}
\hline \multicolumn{4}{|c|}{ Linhagem $\mathrm{BH}$} & \multicolumn{2}{|c|}{ Linhagem SJ } \\
\hline $\begin{array}{l}\text { Total de } \\
\text { vermes por } \\
\text { camundongo } \\
\text { (X) }\end{array}$ & $\begin{array}{l}\text { No de } \\
\text { granulomas } \\
\text { por verme } \\
\text { (Y) }\end{array}$ & $\begin{array}{c}\text { Valores } \\
\text { ajustados } \\
\text { para } \mathrm{Y} \\
\left(\mathrm{Y}_{\mathrm{T}}\right)\end{array}$ & $\begin{array}{l}\text { Total de } \\
\text { vermes por } \\
\text { camundongo } \\
\text { (X) }\end{array}$ & $\begin{array}{c}\text { No de } \\
\text { granulomas } \\
\text { por verme } \\
\text { (Y) }\end{array}$ & $\begin{array}{c}\text { Valores } \\
\text { ajustados } \\
\text { para } \mathrm{Y} \\
\left(\mathrm{Y}_{\mathrm{T}}\right)\end{array}$ \\
\hline 1 & 879 & 736 & 14 & 11 & 11 \\
\hline 3 & 337 & 417 & 3 & 51 & 51 \\
\hline 3 & 350 & 417 & 5 & 31 & 31 \\
\hline 3 & 350 & 417 & 3 & 113 & 107 \\
\hline 3 & 417 & 417 & 4 & 101 & 90 \\
\hline 4 & 346 & 359 & 5 & 83 & 79 \\
\hline 4 & 387 & 359 & 19 & 32 & 36 \\
\hline 5 & 343 & 320 & 19 & 46 & 36 \\
\hline 6 & 309 & 210 & 6 & 238 & 247 \\
\hline 8 & 232 & 251 & 7 & 213 & 235 \\
\hline 6 & 319 & 210 & 10 & 166 & 207 \\
\hline 6 & 326 & 210 & 10 & 171 & 207 \\
\hline 12 & 173 & 203 & 9 & 195 & 215 \\
\hline 6 & 357 & 210 & 15 & 144 & 180 \\
\hline 10 & 215 & 223 & 11 & 250 & 201 \\
\hline 11 & 204 & 212 & 20 & 155 & 163 \\
\hline 11 & 211 & 212 & 10 & 319 & 207 \\
\hline 12 & 193 & 203 & 19 & 168 & 166 \\
\hline 14 & 179 & 187 & 20 & 163 & 163 \\
\hline 14 & 190 & 187 & 22 & 165 & 158 \\
\hline 15 & 195 & 181 & 22 & 164 & 158 \\
\hline 16 & 187 & 148 & 30 & 147 & 142 \\
\hline 17 & 181 & 169 & 34 & 141 & 136 \\
\hline- & - & - & 44 & 141 & 124 \\
\hline
\end{tabular}

animais infectados pela linhagem paulista, parece ter havido uma compensação nos resultados finais, traduzida en indices de mortalidade semelhantes.

Com efeito, encontramos nos camundongos infectados com a linhagem paulista, aproximadamente o dobro de vermes desenvolvidos em camundongos infectados com a linhagem mineira (Tabela 2). Acreditamos que a menor destruição dos esquistossômulos da linhagem paulista seja resultado de uma menor mobilização de defesa orgânica, por parte do hospedeiro, possivelmente relacionada con a patogenicidade atenuada do parasita.

Obtivemos numeros semelhantes de granulomas hepáticos nos vários lotes de camundongos perfundidos, embora os valores referentes à relação granuloma/verme, da linhagem mineira, tenham sido muito superiores aos verificados na linhagem paulista (Tabelas 3, 4, 5, 6 e 7). Esta diferença dos valores representativos da relação granuloma/verme foi atenuada, se considerarmos somente os vermes fêmeas. Segundo Bogliolo2 (1959) os ovos fecun- 
LEMOS NETO, R. C. et al. Alguns aspectos referentes ao estudo de linhagens de Schistosoma mansoni Sambon, 1907, provenientes dos Estados de Minas Gerais e de São Paulo, Brasil. Rer. Saúde pübl., S. Paulo, 12:277-90, 1978.

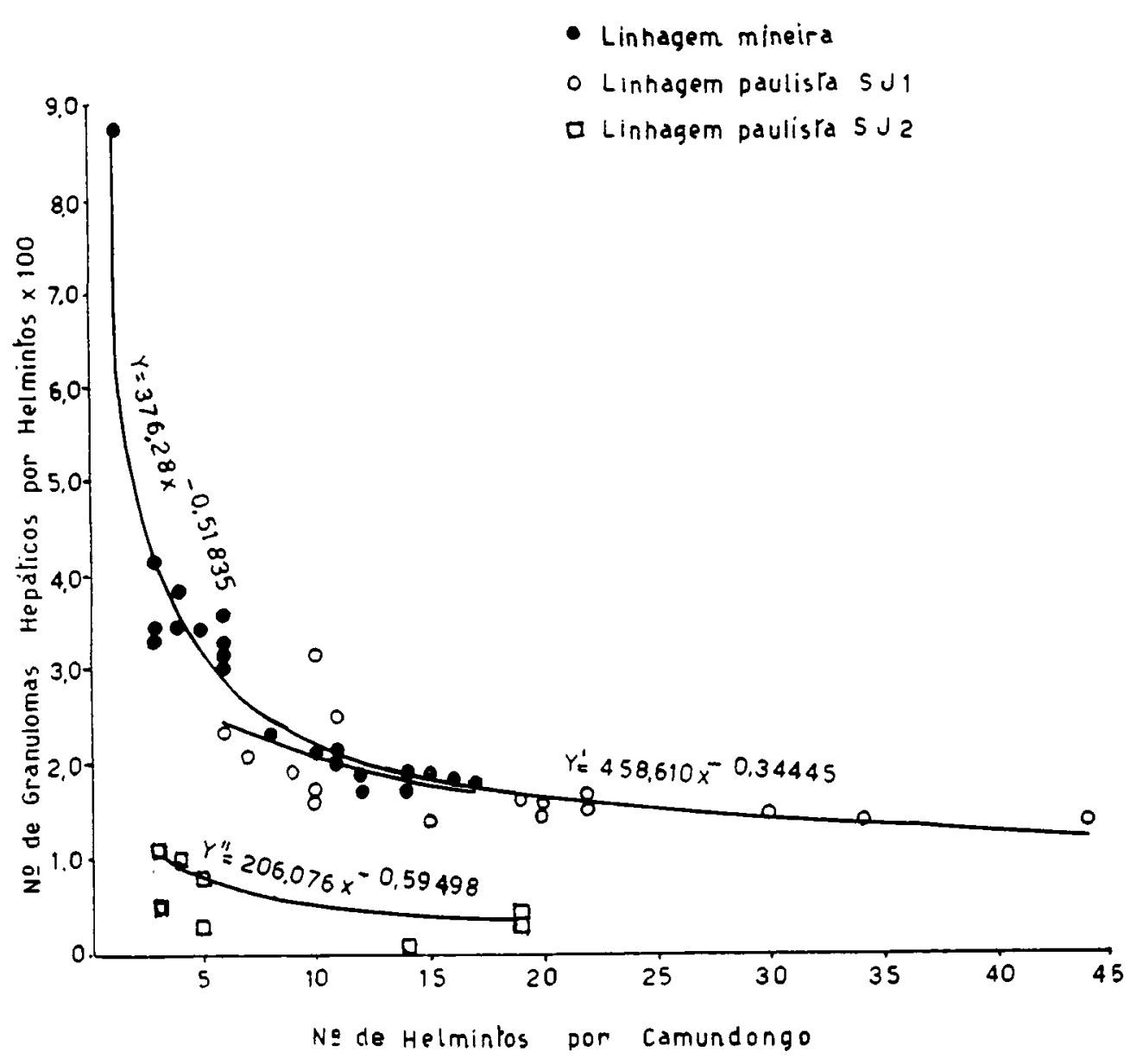

Fig. 4 - Relação entre o número de granulomas hepáticos por vermes adultos e o número de vermes adultos das linhagens $\mathrm{BH}$ e $\mathrm{SJ}$ encontrados em Mus musculus.

dados são mais patogênicos. Desta forma a quantidade de vermes machos teria importância na patogenicidade da infecção, sendo mais expressivo referir-se à relação granuloma/vermes totais.

Acreditamos que o número semelhante de granulomas observado nas duas linhagens tenha sido a causa dos indices de mortalidade (também semelhantes) nos dois grupos de camundongos.

Ao estudarmos as funções que exprimem a correlação granulomas hepáticos-número de vermes, verificamos haver paralelismo entre as duas linhagens. Esse paralelismo, observado na Fig. 1, foi comparado estatisticamente. Os coeficientes de correlação (r) encontrados para a relação granuloma verme, significam que, tanto na linhagem $\mathrm{BH}$ como SJ, o aumento de granulomas hepáticos foi proporcional ao aumento do número de vermes.

Esta representação não é, entretanto, coerente com o fenômeno biológico, porquanto fornece valores significativos para 
LEMOS NETO, R. C. et al. Alguns aspectos referentes ao estudo de linhagens de Schistosoma mansoni Sambon, 1907, provenientes dos Estados de Minas Gerais e de São Paulo, Brasil. Rev. Saúde públ., S. Paulo, 12:277-90, 1978.

\section{TABELA 8}

Viabilidade de penetração de cercárias de $\mathbf{S}$. mansoni, das linhagens de BH e SJ, pelo tegumento da cauda de camundongos albinos.

\begin{tabular}{|c|c|c|c|}
\hline \multicolumn{3}{|c|}{ Linhagens } & (SJ) \\
\hline $\begin{array}{l}\text { Cercárias que } \\
\text { penetraram }\end{array}$ & $\begin{array}{l}\text { Cercárias que } \\
\text { não penetraram }\end{array}$ & $\begin{array}{l}\text { Cercárias que } \\
\text { penetraram }\end{array}$ & $\begin{array}{l}\text { Cercárias que } \\
\text { não penetraram }\end{array}$ \\
\hline 180 & 20 & 160 & 40 \\
\hline 182 & 18 & 166 & 34 \\
\hline 181 & 19 & 160 & 40 \\
\hline 184 & 16 & 168 & 32 \\
\hline 180 & 20 & 164 & 36 \\
\hline 192 & 8 & 166 & 34 \\
\hline 186 & 14 & 162 & 38 \\
\hline 184 & 16 & 168 & 32 \\
\hline 184 & 16 & 156 & 44 \\
\hline 194 & 6 & 168 & 32 \\
\hline 189 & 11 & 155 & 45 \\
\hline 190 & 10 & 152 & 48 \\
\hline 184 & 16 & 146 & 54 \\
\hline 190 & 10 & 158 & 42 \\
\hline 176 & 24 & 142 & 58 \\
\hline Total 2776 & 224 & 2391 & 609 \\
\hline Média 185,06 & 14,93 & 159,4 & 40,6 \\
\hline
\end{tabular}

T A B E L A 9

Dados transformados pela fórmula $\alpha^{0}=$ arco seno $\sqrt{P}$ referente às cercárias que não penetram pelo tegumento da cauda de Mus musculus.

\begin{tabular}{|c|c|c|c|}
\hline $\begin{array}{l}\text { Cercárias } \mathrm{BH} \\
\text { que não } \\
\text { penetraram } \\
(\%)\end{array}$ & $\begin{array}{l}\text { Cercárias } S J \\
\text { que não } \\
\text { penetraram } \\
(\%)\end{array}$ & \multicolumn{2}{|c|}{$\begin{array}{l}\text { Transformação das percentagens } \\
\text { em ângulos } \alpha^{\circ}=\text { arco seno } \sqrt{\mathrm{P}}\end{array}$} \\
\hline 10,0 & 20,0 & 18,43 & 26,57 \\
\hline 9.0 & 17,0 & 17,47 & 24,35 \\
\hline 9,5 & 20,0 & 17,95 & 26,57 \\
\hline 8,0 & 16,0 & 16,43 & 23,58 \\
\hline 10,0 & 18,0 & 18,43 & 25,10 \\
\hline 4,0 & 17,0 & 11,54 & 24,35 \\
\hline 7,0 & 19,0 & 15,34 & 25.84 \\
\hline 8,0 & 16,0 & 16,43 & 23,58 \\
\hline 8,0 & 22,0 & 16,43 & 27,97 \\
\hline 3,0 & 16,0 & 9,97 & 23,58 \\
\hline 5,5 & 22,5 & 13,56 & 28,32 \\
\hline 5,0 & 24,0 & 12,92 & 29,33 \\
\hline 8,0 & 27,0 & 16,43 & 31,31 \\
\hline 5,0 & 21,0 & 12,92 & 27,27 \\
\hline 12,0 & 29,0 & 20,27 & 32,58 \\
\hline 112,5 & 304,5 & 334,52 & 400,30 \\
\hline
\end{tabular}




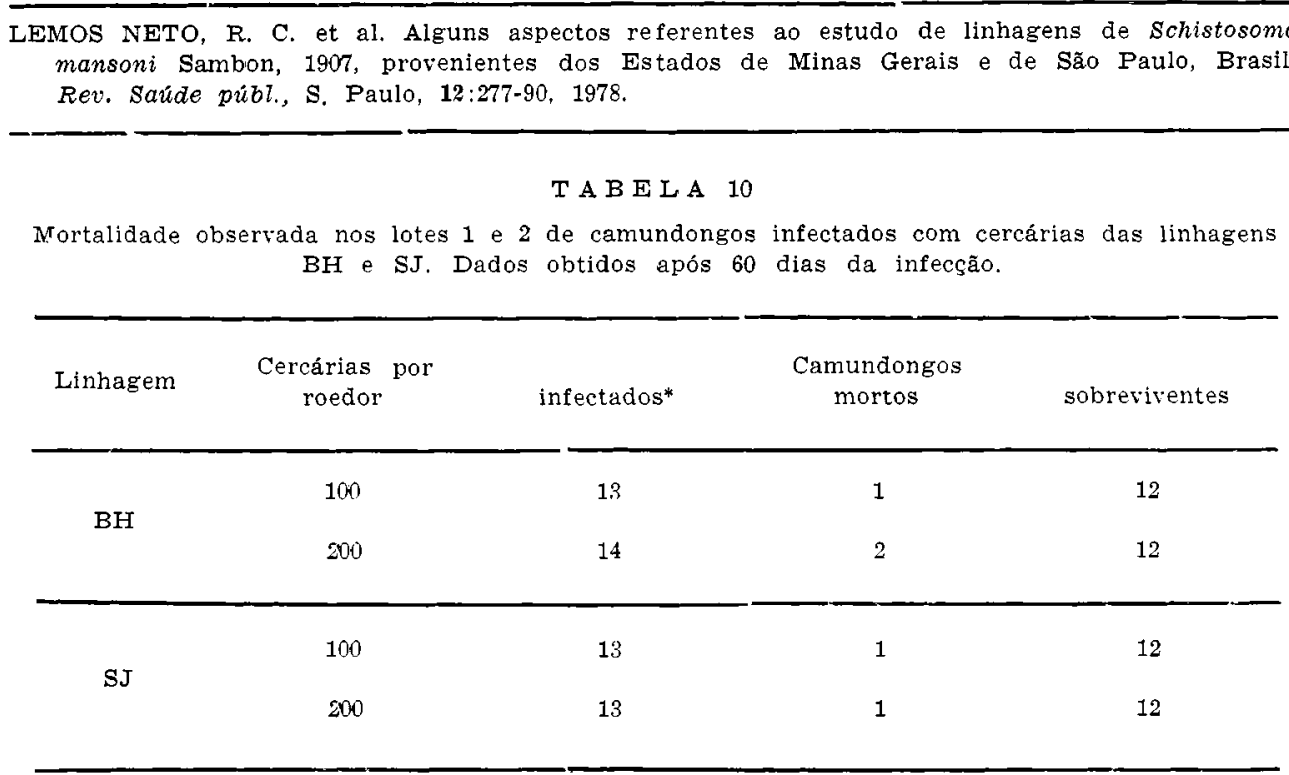

* animais nos quais foi constatada a infeccão.

$\mathrm{y}$ quando $\mathrm{x}=0$, pressupondo a existência de lesões na ausência de infecção. Nessas circunstâncias, o ajustamento hiperbólico é mais adequado a representação do fenômeno, pois seus valores partem da origem $\mathrm{G}=0$ quando $\mathrm{x}=0$ (Figs. 2 e 3 ).

Analisando as Figs. 2 e 3 verificamos que os valores da função $\mathrm{GH}=\mathrm{f}(H M)$, onde $\mathrm{GH}$ é granuloma hepático e $\mathrm{HM}$ número de esquistossomos, apresentaram-se menos comportados na linhagem paulista do que na linhagem mineira.

A análise da Figura 4 onde está colocada na ordenada o número de granulomas hepáticos por verme e na abscissa o número de vermes por camundongo, permite-nos formular a hipótese da existência de duas sub-populações de $S$. mansoni da linhagem (SJ1 e SJ2). Esta hipótese encontra apoio nos trabalhos de Bastos 1 (1975) que demonstrou a existência de diferenças no comportamento biológico entre a linhagem isolada de fezes humanas de doentes autóctones e a isolada de roedores silvestres naturalmente infectados.

Verificamos, nas duas linhagens, que quanto maior o número de vermes por camundongo, menor o número de granulomas hepáticos por verme, isto é, quanto maior a densidade populacional de $S$. mansoni, menor a formação de granulomas hepáticos por verme (Fig. 4).

$\mathrm{Na}$ determinação do poder de penetração das cercárias (Tabela 8 ) verificamos maior capacidade por parte das larvas da linhagem mineira. Estes resultados estão de acordo com o trabalho realizado por Magalhães e Carvalho $^{i}$ (1969), embora estes autores não encontrassem diferenças significativas quanto ao número de vermes adultos que se desenvolveram nos hospedeiros definitivos após a penetração das cercárias (Magalhães e Carvalho ${ }^{8}$, 1973). 
LEMOS NETO, R. C. ct al. Alguns aspectos referentes ao estudo de linhagens de Schistosoma mansoni Sambon, 1907, provenientes dos Estados de Minas Gerais e de São Paulo, Brasil. Rer. Saúde pübl, S. Paulo, 12:277-90, 1978.

LEMOS NETO, R. C. et al. [Some aspects of Schistosoma mansoni strains originating from the States of Minas Gerais and S. Pallo, Brazil.] Rev. Saude públ., S. Pallo, 12:277-90, 1978.

ABSTRACT: Some of the behavioral aspects of the Minas Gerais and S. Paulo states' strains Schistosoma mansoni, concerning the penetration capability of cercariae of each strain and the relationship between hepatic granulomata and worms, were studied. The Minas Gerais' strain cercariae present more penetration capability and the ratio between the numbers of hepatic granulomata and number of worms varied inversely with the number of infecting worms per mouse, in both strains.

UnITERMS: Schistosoma mansoni, strains. Schistosomiasis.

\section{REFERENCIAS BIBLIOGRAFICAS}

1. BASTOS, O. C. Estudo do comportamento parasitológico e imunológico das linhagens humana e silvestre do Schisto. soma mansoni Sambon, 190\%. Campinas, 1975. [Dissertação de Mestrado - Instituto de Biologia da UNICAMP]

2. BOGLIOLO, L. Esquistossomose: patologia. Rev. bras. Malar, 11:359-424, 1959.

3. BRENER, Z, et al. Terapêutica experimental da esquistossomose mansoni. Aplicação do método de isolamento de granulomas do fígado de camunlongos. Rev. bras. Malar, 8:583-7, 1956.

4. FISHER, R. H. \& YATES, F. Tabelas estatisticas para pesquisa em biologia, medicina e agricultura. São Paulo, Polígono/Ed. USP, 1971. p. 78-9.

5. HILL, J. Chemotherapeutic studies with laboratory infections of Schistosoma mansoni. Ann. trop. Med. Parasit. $\mathbf{5 0}: 39-48, \mathbf{1 9 5 6 .}$

6. MAGALhÃes, L. A. Técnica para avaliacão da viabilidade de penetração de cercárias de Schistosoma mansoni em Mus musculus. Hospital, Rio de $\mathrm{Ja}$ neiro, 75:137-40, 1969.

7. MAGALhāes, L. A. \& CARVALHo, J. F. Determinação do número de cercárias provenientes de cepas diferentes de Schistosoma mansoni que conseguem penetrar, sob determinadas condiçoes de laboratório, em Mus musculus. Rev. Soc. bras. Med. trop., 3:249-51, 1969.

MAGALHÃE, L. A. \& CARVALHO, J. F. Desenvolvimento do $S$. mansoni das linhagens de Belo Horizonte (MG) e de são José dos Campos (SP) em Mus musculus. Rev. Saude puibl., $\mathbf{S}$. Paulo, 7:285-7, 1973.

9. İIAGALHÃES, L. A. \& CARVALHO, J. F. Estudo morfológico de Schistosoma mansoni pertencentes a linhagens de Belo Forizonte ( $M G$ ) e de São José dos Campos (S.P.), Rev. Saúde públ., S. Paulo, i:289-94, 1973.

10. MAGALhäS, L. A. \& CARVALHO, J. F. Sobre o comportamento de duas linhagens de Schistosoma mansoni Sambon, 1907. Proposıção para método de estudo quantitativo. Rev. Soc. bras. Med. trop., 10:169-94, 1976.

11. MAGALHãES, L. A. et al. Alguns dados referentes ao estudo parasitológico e anatomopatológico de duas linhagens de Schistosoma mansoni Sambon, 1907. Rev. Satide puibl., S. Paulo, 9:1-5, 1975.

12. PARAENSE, W. L. \& CORREA, L. R. Sobre a ocorrência de duas raças biológicas do Schistosoma mansoni no Brasil. Cienc. Cult., 15:245-6, 1963. [apresentado à 15a. Reunião Anual da Sociedade Brasileira para o Progresso da Ciência, Campinas, 1963]

13. PELLEGRINO, J. \& BRENER, Z. Method for isolating Schistosome granulomas from mouse liver. J. Parasit., 42:564, 1956.

14. PELlEGRINO, J. \& MACEDO, D. G. A simplified method for the concentration of cercariae. J. Parasit., 41:32930,1955 
LEMOS NETO, R. C. et al. Alguns aspectos referentes ao estudo de linhagens de Schistosoma mansoni Sambon, 1907, provenientes dos Estados de Minas Gerais e de São Paulo, Brasil. Rev. Saúde publ., S. Paulo, 12:277-90, 1978

15. PiedrabUena, A. E. \& BARACHO, I. R. Teste de significância entre os coeficientes de correlação. Cienc. Cult., S. Paulo, 28:191-2, 1976.

16. STANDEN, O. D. Experimental schistosomiasis. III. Chemotherapy and mode of drug action. Anr. trop. Med. Parasit., $4 \mathbf{7}: 26-43,1953$.
17. YOLLES, T. K, et al. A technique for the perfusion of laboratory animals for the recovery of Schistosomes, J. Parasit., $33: 419-26, \quad 1947$.

Recebido para publicą̧ão em 09/11/197\%

Aprovado para publicạ̄ão em 13/04/19\%8

\section{NOTICIĀRIO}

\section{CONGRESSO DA SOCIEDADE BRASILEIRA DE MEDICINA TROPICAL}

\section{Campinas, SP - 4 a 8 de fevereiro de 1979.}

Como o apoio da Universidade Estadual de Campinas (UNICAMP) será realizado em 1979 o Congresso supramencionado.

Com a colaboração das Comissões Executiva e Científica, foi estabelecido o programa do Congresso que se desenvolverá por meio de:

"Forum" para análise de questōes medico sociais, que será constituido de quatro sessões, referentes aos temas: "O ensino de clinica das doenças transmissiveis, em niveis de graduação e pós-graduação, no Brasil"; "Saúde e desenvolvimento, no Brasil. 1 - Expansão demográfica e doenças transmissiveis"; "Saúde e desenvolvimento, no Brasil. II - Grandes endemias: importância, combate e aspectos ligados ao trabalho de indivíduos acometidos; migração populacional interna"; "Doenças transmissiveis e assistência médica, no Brasil".

Conferências, em número de cinco, referentes aos temas: "Progressos nacionais relativos às doenças transmissiveis, em
1978"; "Progressos internacionais às doenças transmissíveis, em 1978"; "Progressos recentes, de natureza imunológica, relativos às doenças parasitárias"; "Concepções atuais sobre epidemiologia e controle da esquistossomose mansônica"; "Progressos relativos às doenças transmissiveis, distinguidos em comunicações científicas apresentadas no XV Congresso da Sociedade Brasileira de Medicina Tropical".

Temas Livres, com apresentação de comunicações científicas, em sessões tradicionais ou através de "posters".

Cursos, em número de três, referentes a "Antibioticoterapia", "Imunizações" e "Doença de Chagas". Os dois primeiros procurarão promover atualização e, o outro, terá o intuito de salientar pesquisas de expressivo nível, realizada por cientistas brasileiros.

Os pedidos de informação e inscrições devem ser dirigidos ao DOC - Associação Médica Brasileira - Av. Paulista, 1159, ci 314/315 - São Paulo, SP - Telefone 287-3081. 